Revue internationale P.M.E.

Économie et gestion de la petite et moyenne entreprise

\title{
L'influence de la taille organisationnelle sur le plafonnement de carrière
}

\section{Serge Beauchamp et Bruno Fabi}

Volume 6, numéro 2, 1993

URI : https://id.erudit.org/iderudit/1008211ar

DOI : https://doi.org/10.7202/1008211ar

Aller au sommaire du numéro

Éditeur(s)

Presses de l’Université du Québec

ISSN

0776-5436 (imprimé)

1918-9699 (numérique)

Découvrir la revue

Citer cet article

Beauchamp, S. \& Fabi, B. (1993). L'influence de la taille organisationnelle sur le plafonnement de carrière. Revue internationale P.M.E., 6(2), 83-107. https://doi.org/10.7202/1008211ar

\section{Résumé de l'article}

Le présent article traite de l'influence de certaines caractéristiques organisationnelles sur le plafonnement de carrière, une attention particulière étant apportée à l'influence de la taille de l'organisation. Pour y parvenir, une démarche empirique a été effectuée auprès d'une population de 441 cadres intermédiaires provenant des 14 centres de services sociaux (CSS) du Québec.

Les résultats révèlent que les répondants œuvrant au sein des organisations de dimension moins importante s'avèrent plus sujets au plafonnement de carrière que leurs collègues œuvrant dans des organisations de plus grande dimension. D'autres résultats font ressortir l'absence d'influence significative de la structure organisationnelle sur le plafonnement de carrière, ce dernier phénomène s’avérant par ailleurs influencé par la nature du poste occupé. À cet effet, on relève que les cadres œuvrant au sein d'un service conseil semblent plus sujets au plafonnement de carrière que leurs collègues des services hiérarchiques. Finalement, en plus de vérifier l'effet de certaines caractéristiques individuelles sur le plafonnement de carrière, cette étude a contribué à démontrer les limites de l'approche traditionnelle consistant à mesurer la variable dépendante à partir de seuils arbitraires d'ancienneté dans le poste actuel. Nos analyses suggèrent en effet la pertinence de délaisser une telle approche dichotomique pour adopter une mesure continue, plus flexible et moins arbitraire. L'ensemble de ces résultats fait l'objet d'une discussion relative aux implications d'une gestion renouvelée du cheminement de carrière dans les organisations, particulièrement dans les PME.
Ce document est protégé par la loi sur le droit d'auteur. L'utilisation des services d’Érudit (y compris la reproduction) est assujettie à sa politique d'utilisation que vous pouvez consulter en ligne.

https://apropos.erudit.org/fr/usagers/politique-dutilisation/ 


\title{
L'influence de la taille organisationnelle sur le plafonnement de carrière ${ }^{1}$
}

\author{
Serge BEAUCHAMP* \\ Centre des services sociaux du Centre du Québec \\ Bruno $\left.F A B\right|^{\star *}$ \\ Université du Québec à Trois-Rivières
}

\begin{abstract}
RÉSUMÉ
Le présent article traite de l'influence de certaines caractéristiques organisationnelles sur le plafonnement de carrière, une attention particulière étant apportée à l'influence de la taille de l'organisation. Pour y parvenir, une démarche empirique a été effectuée auprès d'une population de 441 cadres intermédiaires provenant des 14 centres de services sociaux (CSS) du Québec.

Les résultats révèlent que les répondants œuvrant au sein des organisations de dimension moins importante s'avèrent plus sujets au plafonnement de carrière que leurs collègues ceuvrant dans des organisations de plus grande dimension. D'autres résultats font ressortir l'absence d'influence significative de la structure organisationnelle sur le plafonnement de carrière, ce dernier
\end{abstract}

1. Les auteurs aimeraient remercier le professeur Bruno Rémillard pour ses recommandations relatives à certaines analyses statistiques. Il convient également de remercier les trois lecteurs anonymes pour leurs commentaires. Toutes les erreurs présentes dans cet article seraient toutefois la responsabilité entière des auteurs.

* Serge Beauchamp est détenteur d'une M.Sc. de l'Université du Québec à TroisRivières. Il est directeur des services administratifs au Centre de services sociaux du Centre du Québec. Adresse : Centre des services sociaux du Centre du Québec, 2700, boul. des Forges, Trois-Rivières (Québec), G8Z 1V2.

** Bruno Fabi est professeur agrégé en Gestion des ressources humaines à l'Université du Québec à Trois-Rivières. Il est détenteur d'un doctorat en psychologie industrielle/organisationnelle de l'Université de Montréal. Ses articles ont paru dans divers périodiques dont International Journal of Quality and Reliability Management, International Journal of Project Management, Revue Canadienne des Sciences de l'Administration, Psychological Reports, Educational and Psychological Measurement et Australian Bulletin of Labour. Le professeur Fabi est membre du Groupe de recherche en économie et gestion des PME. Adresse : GREPME, Université du Québec à Trois-Rivières, C.P. 500, TroisRivières (Québec) G9A 5H7. 
phénomène s'avérant par ailleurs influencé par la nature du poste occupé. $\grave{A}$ cet effet, on relève que les cadres œuvrant au sein d'un service conseil semblent plus sujets au plafonnement de carrière que leurs collègues des services hiérarchiques. Finalement, en plus de vérifier l'effet de certaines caractéristiques individuelles sur le plafonnement de carrière, cette étude a contribué à démontrer les limites de l'approche traditionnelle consistant à mesurer la variable dépendante à partir de seuils arbitraires d'ancienneté dans le poste actuel. Nos analyses suggèrent en effet la pertinence de délaisser une telle approche dichotomique pour adopter une mesure continue, plus flexible et moins arbitraire. L'ensemble de ces résultats fait l'objet d'une discussion relative aux implications d'une gestion renouvelée du cheminement de carrière dans les organisations, particulièrement dans les PME.

\section{ABSTRACT}

This paper looks at the influence of a number of organizational attributes on career plateauing, paying special attention on the influence of organizational size. An empirical process was used on a sample of 441 middle managers in Quebec's 14 Social Service Centers (SSC). The results show that respondents working in smaller organizations are more likely to experience career plateauing than their colleagues in larger organizations. Other results reveal that organizational structure has no significant influence on career plateauing and, in contrast, that the type of position held does have an influence. Managers in staff departments seem more subject to plateauing than those in line departments. Finally, in addition to examining the effect of some individual attributes on career plateauing, the research helps illustrate the limitations of the traditional approach of measuring dependent variables on the basis of arbitrary thresholds of lenght of time in the present position. Our analyses suggest that this kind of dichotomic approach should be abandoned in favor of a continuous, more flexible and less arbitrary measurement. The implications of the overall results in terms of renewed management of career paths in organizations in general, and SMEs in particular, are discussed.

\section{RESUMEN}

Este articulo analiza la influencia de ciertas caractérísticas organizacionales sobre el estancamiento de carrera, una atención particular ha sido dada a la influencia del tamano organizacional. Para lograrlo, una investigación empírica se realizó con una población de 441 funcionarios de nivel intermedio, provenientes de 14 Centros de Servicio Social (CSS) de Quebec.

Los resultados revelan que los empleados que se desempeñan al interior de organizaciones de menor dimensión, se muestran mas sujetos al estancamiento de carrera que sus colegas que trabajan en organizaciones de dimensión mayor.

Otros resultados sacan a relucir la no influencia de la estructura organizacional sobre el estancamiento de carrera, este último fenomeno resulta por otro lado, influenciado por la naturaleza del puesto ocupado. Al respecto, se revela que los funcionarios que se desempeñan al interior de un servicio de consejería se ven más sujetos al estancamiento de carrera que sus colegas de servicios jerarquicos. 
Finalmente, ademas de verificar el efecto de ciertas características individuales sobre el estancamiento de carrera, este estudio contribuye a demostrar las limitaciones del enfoque tradicional consistente en medir la variable dependiente a partir de niveles artibrarios de antiguedad en el puesto actual. Nuestros analisis sugieren en efecto la pertinencia de abandonar ese enfoque dicotómico, para adoptar una medida continua, mas flexible y menos arbitraria. El conjunto de estos resultados es el objeto de una discusión relativa a las implicasiones de una gestión renovada de desarrollo de carrera en las organizaciones, particularmente en la PME.

\section{Introduction}

Les environnements interne et externe des organisations sont en profonde mutation. Ces dernières, les PME comme les plus grandes, se voient confrontées à de nouvelles tendances lourdes qui affecteront inévitablement leur orientation stratégique et, par voie de conséquence, leurs pratiques de gestion des ressources humaines. On pense ici à des tendances telles que la globalisation et la déréglementation des marchés, l'intensification de la concurrence, la constitution de marchés communs, l'augmentation des acquisitions et des fusions, l'implantation croissante des nouvelles technologies, la différenciation dans la consommation, de même que l'évolution rapide des valeurs et des comportements sociaux (Audet, 1990; Bourbonnais et Gosselin, 1988). Sur le plan organisationnel, on observe également une évolution relative à certaines caractéristiques de la main-d'œuvre: vieillissement, diversification ethnique, féminisation, augmentation de la scolarité, modification des attentes à l'égard du travail, prolifération des couples à double carrière (Cardinal et Lamoureux, 1992; Fabi, 1992).

Il va sans dire que les organisations publiques ne peuvent échapper à l'influence de ces turbulences fondamentales. C'est ainsi que l'on assiste graduellement à la réduction de la taille de fonctions publiques et au désengagement de l'État dans de nombreux secteurs de l'économie. On n'a ici qu'à penser aux nombreuses privatisations, ainsi qu'aux impératifs croissants d'autofinancement auxquelles font face ces organisations. Comme le soulignent Tremblay et Roger (1992), la gestion du secteur public sera désormais soumise aux contraintes de la concurrence. En contexte québécois, cette évolution a amené certains auteurs à proposer une réforme des politiques sociales afin de limiter les coûts de l'État-providence (Beausoleil, 1987). Dans cette foulée, une commission d'enquête a été mise sur pied afin de diagnostiquer et d'améliorer le système québécois de santé et des services sociaux (Rochon, 1988). Or, la réforme d'un tel réseau constitue un défi majeur pour les organisations concernées, notamment en ce qui a trait à l'efficience dans l'utilisation des ressources (Beauchamp et Fabi, 1992). Plus spécifiquement, dans une perspective de développement des ressources humaines, des mesures sont mises de l'avant relativement à la mobilité et à l'orientation de carrière du personnel cadre. 
Cette dernière problématique a intéressé quelques chercheurs, certains se concentrant sur l'absence de mobilité parfois associée au plafonnement de carrière. Ainsi, plusieurs études empiriques ont permis d'établir des relations entre différentes caractéristiques individuelles et le phénomène de plafonnement de carrière. C'est le cas notamment pour l'âge, le sexe, la scolarité, la performance, la carrière antérieure, l'ancienneté dans l'établissement ainsi que la satisfaction, l'attitude et le comportement au travail.

Par contre, très peu d'études se sont attardées aux caractéristiques organisationnelles susceptibles d'influencer le plafonnement de carrière. Ces études ont tout de même permis d'illustrer les effets qu'ont la taille, la structure organisationnelle, le contexte du travail ainsi que les différentes politiques ou pratiques de dotation de personnel, sur le plafonnement de carrière.

Deux courants majeurs ont marqué la recherche consacrée à ce phénomène. Le premier se caractérise par l'adoption d'une définition objective du concept de plafonnement de carrière basée sur l'ancienneté dans le poste actuel. À l'opposé, le second adopte une définition plutôt subjective du concept faisant appel à la perception ou au sentiment des sujets à l'égard du plafonnement de carrière. La présente étude s'inscrit dans le premier courant de recherche. Il s'agit de poursuivre l'exploration de cette problématique en se concentrant sur l'influence de diverses caractéristiques organisationnelles, notamment la taille des organisations, sur le phénomène de plafonnement de carrière.

\section{Fondements théoriques de la recherche}

\subsection{Définition du concept de plafonnement de carrière}

Il n'existe pas de définition universelle du plafonnement de carrière (Tremblay, 1992). Cependant, plusieurs auteurs définissent ce concept comme étant l'absence de mobilité latérale ou verticale durant une période donnée, laquelle varie de 5 à 10 ans selon les auteurs (Warren et al., 1977; Veiga, 1981; Slocum et al., 1985 ; Gerpott et Domsh, 1987; Tremblay et Roger, 1991). D'ailleurs, un fort consensus semble s'établir autour de l'ancienneté dans le poste actuel comme indicateur de plafonnement de carrière.

Compte tenu du courant de recherche dans lequel s'inscrit cette étude, nous avons retenu la définition de Veiga (1981) comme définition opérationnelle du concept de plafonnement de carrière, soit le «point où la mobilité de carrière future, incluant les mouvements latéraux et verticaux, est peu probable à cause de la durée de séjour prolongée sur le poste actuel». 


\subsection{Modèle conceptuel du plafonnement de carrière}

Les études portant spécifiquement sur le plafonnement de carrière sont relativement peu nombreuses, ce phénomène n'étant l'objet de recherches que depuis la fin des années 70 . Elles ont cependant permis d'examiner certaines causes individuelles et organisationnelles du plafonnement de carrière.

Quelques études significatives ont permis d'établir directement ou indirectement des relations entre certaines caractéristiques organisationnelles et le plafonnement de carrière. Elles ont contribué à faire ressortir des relations significatives au niveau des variables comme la taille de l'organisation (Dalton et Kesner, 1983; Stumpf et Hartman, 1984; Near, 1985; Grand-Rose et Portwood, 1988), la structure organisationnelle (Spilerman, 1977; Vardi, 1980; Granjean, 1981, Scholl, 1983) ainsi que la nature du poste occupé (Spilerman, 1977; Vardi, 1980; Nystrom, 1986).

Par ailleurs, plusieurs études ont permis d'établir des relations entre certaines caractéristiques individuelles et le plafonnement de carrière. À l'instar des études énoncées au paragraphe précédent, le concept de plafonnement de carrière était mesuré objectivement en fonction de l'ancienneté dans le poste actuel. Elles ont ainsi fait ressortir des relations au niveau des variables comme l'âge (Veiga, 1981; Gould et Penley, 1984; Tremblay et Roger, 1991), la scolarité (Gerpott et Domsh, 1987; Tremblay et Roger, 1991), la performance (Veiga, 1981 ; Carnazza et al., 1981 : Slocum et al., 1985 ; Near, 1985 ; Gerpott et Domsh, 1987; Tremblay et Roger, 1991) ainsi que l'ancienneté dans l'organisation (Gould et Penley, 1985; Tremblay et Roger, 1991).

La figure 1 illustre notre modèle conceptuel de la mobilité interne et du plafonnement de carrière. Ce modèle reflète les relations entre, d'une part, les caractéristiques environnementales, individuelles et organisationnelles et, $d$ 'autre part, la mobilité interne ainsi que le plafonnement de carrière, la variable dépendante étant présentée en caractère gras à l'instar des autres variables analysées dans le cadre de cette étude.

\subsubsection{Caractéristiques organisationnelles}

\section{Taille de l'organisation}

Identifiée comme facteur de contingence, la taille de l'organisation influence la structure et la dynamique de l'organisation (Mintzberg, 1982). Dans une étude effectuée auprès de 181 entreprises, Dalton et Kesner (1983) voulaient vérifier le processus de succession à des postes cadres. Leurs résultats montrent que les organisations de plus grande dimension comblent généralement leurs postes de cadres vacants par l'interne. Ces résultats s'expliquent par le fait que ces 
FIGURE 1

Modèle conceptuel de la mobilité interne

et du plafonnement de carrière

(Les variables considérées dans cette étude sont en caractères gras.)

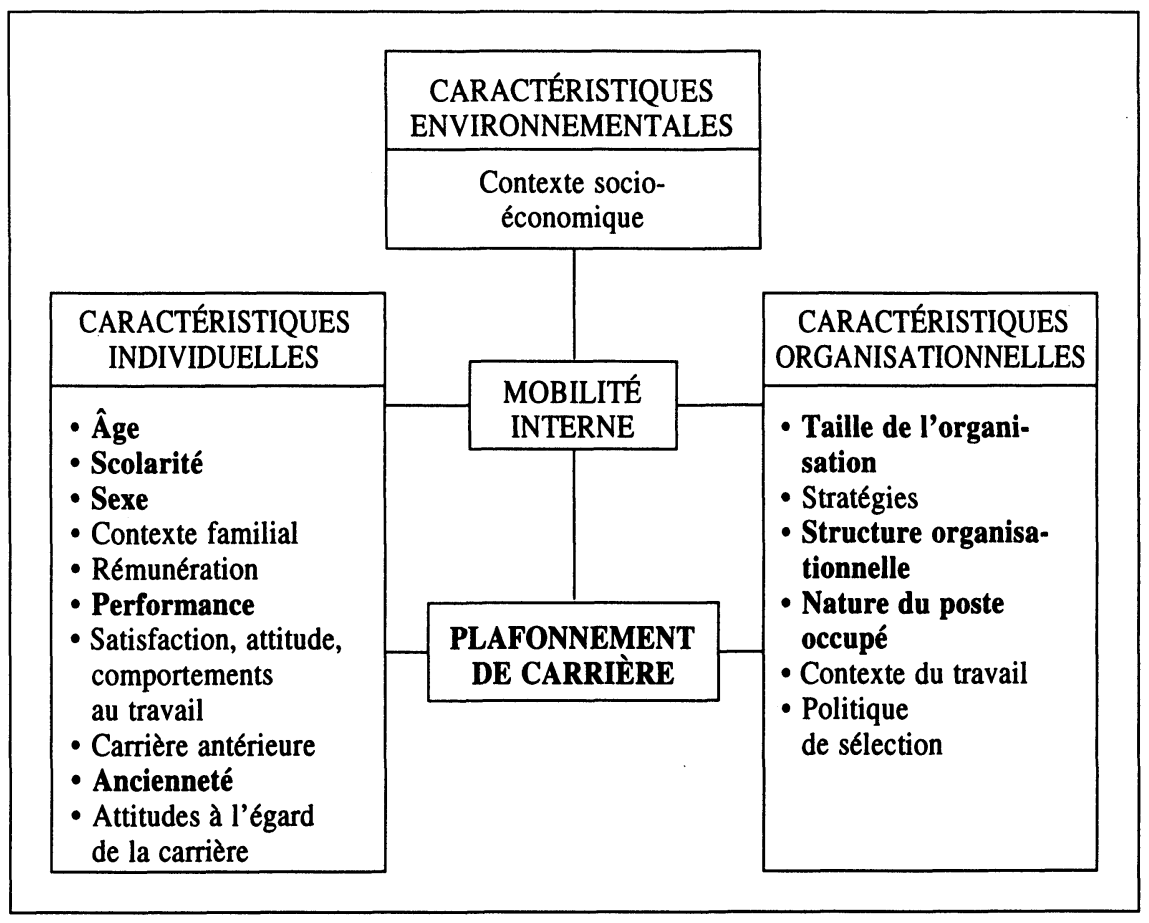

organisations offrent généralement plus de formation à leurs employés, ce qui favorise le développement de leur potentiel et les rend ainsi plus aptes à recevoir de l'avancement ou à assumer un accroissement de responsabilités (Dalton et Kesner, 1983). Dans la même veine, la taille de l'organisation influencerait positivement, selon Stumpf et Hartman (1984), la mobilité et la flexibilité de carrière dans une organisation. Par ailleurs, dans une autre étude portant sur l'appariement des plans individuels de carrière à la gestion de carrière organisationnelle, Gran-Rose et Portwood (1988) trouvent que les organisations de grande dimension possèdent habituellement un système formel de gestion des carrières relié aux activités de planification de l'organisation.

Allant dans le même sens, Near (1985) a établi que les situations de plafonnement de carrière apparaissaient plus fréquemment dans des organisations de dimensions moins importantes. Ces résultats ont été obtenus dans le cadre d'une recherche effectuée auprès d'entreprises œuvrant dans différents secteurs d'activités. Nous pouvons donc concevoir que la dimension d'une 
organisation, conditionnant notamment la structure et les processus, pourra avoir un effet sur les possibilités de mouvements et, par conséquent, le plafonnement de carrière.

\section{Structure organisationnelle}

La structure organisationnelle constitue une autre caractéristique organisationnelle étudiée par les chercheurs. Certaines études ont examiné les relations de cette caractéristique, sous les angles des filières d'emploi et de la nature du poste occupé, avec la mobilité interne et le plafonnement de carrière.

La conception de la structure organisationnelle conditionne notamment les filières d'emplois et le nombre de paliers hiérarchiques à l'intérieur de chacune d'elles. Souvent associée à un service, une filière d'emploi comportant peu de paliers hiérarchiques influencerait négativement les possibilités d'avancement (Spilerman, 1977; Vardi, 1980). En outre, les résultats d'une étude réalisée auprès de différentes institutions fédérales américaines ont démontré que le point d'entrée dans une organisation, compte tenu de la filière d'emploi où il se situe, constitue un prédicteur de mobilité future (Grandjean, 1981).

Par ailleurs, Scholl (1983) suggère une relation négative entre la mobilité interne et le changement de personnel. Cette relation s'explique par le fait que certains cadres qui se retrouvent dans une filière d'emploi sans perspectives d'avancement quittent l'organisation, ce qui a pour effet d'accroître le changement du personnel. Il corrobore ainsi le fait que le nombre de paliers hiérarchiques d'une filière d'emploi définit les limites de la mobilité interne. Par conséquent, des cadres se retrouvant dans une filière d'emploi comportant peu de paliers, ce qui est plus fréquent dans de petites et moyennes organisations, seraient plus susceptibles de vivre une situation de plafonnement de carrière (Tremblay, 1991).

\section{Nature du poste occupé}

L'influence de la nature du poste occupé sur la mobilité interne et le plafonnement de carrière, particulièrement sous l'angle de la fonction, a intéressé certains chercheurs, en particulier Nystrom (1986). S'inspirant du modèle descriptif de l'organisation de Mintzberg, il suggère que les fonctions conseil et hiérarchique peuvent procurer des occasions de carrières différentes. En effet, dans une étude sur 57 cadres, dont 23 occupent une fonction conseil et 34 une fonction hiérarchique, l'auteur a constaté que la mobilité interfonctions s'avère très peu fréquente et non habituelle. De plus, ces résultats illustrent le phénomène de cloisonnement des emplois énoncé par Spilerman (1977), phénomène pouvant expliquer pourquoi certains cadres se retrouvent plus rapidement que d'autres dans une situation de plafonnement de carrière. Il convient toutefois de préciser que ces études concernent spécifiquement la mobilité interfonctions, un concept 
à la fois apparenté mais distinct du problème de plafonnement de carrière à l'intérieur de chacune des fonctions conseil et hiérarchique.

De façon plus pertinente à la présente étude, Tremblay et Roger (1991) ont examiné l'effet de certaines caractéristiques individuelles et organisationnelles sur la stabilité et le plafonnement de carrière. Réalisée auprès de plus de 2000 cadres de plusieurs niveaux (chefs d'unités à directeurs) provenant de trois grands secteurs économiques du Québec (alimentation, pâtes et papiers et fonction publique), leur étude n'a pas permis d'établir de relation significative entre la nature du poste occupé et le phénomène de plafonnement de carrière.

\subsubsection{Caractéristiques individuelles}

\section{Âge}

Certains auteurs ont corroboré les résultats de l'étude de Veiga (1981) relativement à l'âge : les sujets en situation de plafonnement de carrière sont généralement plus âgés, soit en moyenne 45 ans, comparativement aux autres sujets qui ont en moyenne entre 36 et 39 ans (Gould et Penley, 1984; Tremblay et Roger, 1991).

\section{Scolarité}

À l'instar de Near (1983; Tremblay et Roger, 1991) ainsi que de Gerpott et Domsh (1987), d'autres auteurs ont établi que le niveau de scolarité des sujets plafonnés est inférieur aux sujets mobiles (Tremblay et Roger, 1991). Cependant, au sein d'un groupe de sujets homogènes, comme c'est le cas dans l'étude de Gerpott et Domsh (1987) réalisée auprès de 729 sujets travaillant dans des départements de recherche et développement de 11 corporations industrielles, une différence peu significative dans le niveau de scolarité semble subsister entre les sujets réputés en situation de plafonnement de carrière et ceux qui ne le sont pas.

\section{Sexe}

Le sexe constitue une autre caractéristique individuelle pouvant influencer les opportunités de carrière (Grimm et Stern, 1974; Vardi, 1980; Anderson et al., 1981). De même, Michael (1984; Beehr et Juntunen, 1990) relate que les femmes occupent souvent des fonctions conseils, lesquelles sont considérées comme terminales et non comme une étape d'un cheminement de carrière. Selon lui, le réseau de contacts informels dans l'organisation, réseau non également accessible aux hommes et aux femmes, pourrait favoriser le phénomène de plafonnement de carrière chez ces dernières. 


\section{Performance}

Pour certains auteurs, la performance ne constitue pas un facteur distinctif des sujets plafonnés (Carnazza et al., 1981; Slocum et al., 1985; Near, 1985; Tremblay et Roger, 1991). En effet, on a démontré que certains cadres en plafonnement sont aussi performants que d'autres qui ne sont pas en situation de plafonnement de carrière. Pour ces cadres, l'importance que revêt leur travail pour l'organisation et les défis qu'ils y rencontrent s'avèrent suffisamment valorisants pour compenser le besoin d'avancement.

Pour sa part, Veiga (1981), se référant au modèle de Warren et al. (1977), rapporte que le niveau de salaire annuel chez les cadres efficaces est supérieur en moyenne de 3000 dollars à celui des cadres dont le rendement est jugé insatisfaisant, l'augmentation de salaire reflétant généralement l'appréciation de la performance. Dans la même veine, Gerpott et Domsh (1987) ont constaté que les sujets en plafonnement de carrière ont un moins bon rendement que ceux qui sont professionnellement plus mobiles.

Nous constatons donc que la relation entre la performance et le plafonnement de carrière n'est pas aussi claire qu'on pourrait l'imaginer. Des facteurs comme la satisfaction et la motivation au travail influencent certainement la performance d'un individu en situation de plafonnement de carrière.

\section{Ancienneté dans l'organisation}

L'ancienneté dans l'organisation constitue une autre variable dont on a étudié l'effet sur le plafonnement de carrière. À cet effet, il ressort que l'ancienneté semble plus élevée chez les sujets en situation de plafonnement de carrière selon les résultats de quelques études (Gould et Penley, 1985; Tremblay et Roger, 1991). Ces résultats corroborent ceux de Mills (1985) qui avait identifié une relation négative entre l'ancienneté et les possibilités d'avancement. Comme nous pouvons le constater, peu de recherches ont permis de conclure à une certaine relation entre cette variable et le plafonnement de carrière.

\section{Hypothèses}

À la lumière des résultats présentés antérieurement, nous avons formulé trois hypothèses de recherche permettant d'examiner les relations entre, d'une part, la taille de l'organisation, le nombre de paliers hiérarchiques compris dans le service où œuvre le cadre, ainsi que la nature du poste occupé et, d'autre part, l'ancienneté dans le poste actuel. 


\section{Hypothèse 1}

Il y aura une relation négative et significative entre la taille organisationnelle et l'ancienneté dans le poste actuel.

\section{Hypothèse 2}

Il y aura une relation négative et significative entre le nombre de paliers hiérarchiques compris dans le service et l'ancienneté dans le poste actuel.

\section{Hypothèse 3}

Il n'y aura pas de différence significative au niveau de l'ancienneté dans le poste actuel entre les cadres qui occupent un poste conseil et ceux qui occupent un poste hiérarchique.

\section{Méthodologie}

\subsection{Population étudiée et procédure de collecte de données}

Les données recueillies concernent l'ensemble des 441 cadres intermédiaires, titulaires d'un poste dans un des 14 CSS du Québec répartis comme suit:

- 9 cadres intermédiaires dans 1 CSS comptant moins de 100 postes;

- 87 cadres intermédiaires dans 7 CSS comptant entre 101 et 200 postes;

- 46 cadres intermédiaires dans 2 CSS comptant entre 201 et 500 postes;

- 299 cadres intermédiaires dans 4 CSS comptant plus de 500 postes.

La collecte de données a été réalisée à l'aide d'un questionnaire devant être complété par le Service des ressources humaines de chaque CSS. Accompagnant ce questionnaire, une lettre réitérait les objectifs de l'étude et offrait aux répondants un support téléphonique pour pallier, le cas échéant, les difficultés concernant la compréhension du questionnaire. De plus, une enveloppe de retour préadressée et affranchie accompagnait le tout. Le taux de réponse a été de $100 \%$, ce qui implique que nos analyses porteront sur la population des cadres intermédiaires des CSS du Québec.

Les sujets, dont $52 \%$ sont de sexe masculin, sont âgés en moyenne de 45 ans et comptent en moyenne 15 années de services dans l'organisation dont 6 dans leur poste actuel. Presque tous $(92 \%)$ possèdent une formation universitaire de premier cycle, $50 \%$ d'entre eux détenant une maîtrise. De plus, $80 \%$ des cadres occupent une fonction hiérarchique. Finalement, $35 \%$ des sujets se sont vus reconnaître une contribution exceptionnelle, ce qui équivaut à un rendement supérieur dans d'autres contextes organisationnels. 


\subsection{Instruments de mesure}

La taille de l'organisation était mesurée à partir du nombre de postes permanents en équivalence de temps complet. Cette mesure était conforme aux études antérieures où le nombre d'employés était retenu (Near, 1985; Grand-Rose et Portwood, 1988). Certains chercheurs ont retenu en plus du nombre d'employés, le chiffre d'affaires comme mesure de la taille de l'organisation (Dalton et Kesner, 1983). Comme les CSS sont des organismes parapublics entièrement subventionnés par le ministère de la Santé et des Services sociaux (MSSS), et que la structure de coûts est relativement homogène - la masse salariale représentant en moyenne $80 \%$ des coûts d'opération -, il a semblé pertinent de se restreindre au nombre de postes comme mesure de la taille de l'organisation. Par ailleurs, catégoriser les organisations en petite, moyenne et grande dimensions nous aurait exposé à des problèmes de catégorisation arbitraire et aurait donné un niveau de mesure inutilement réductionniste.

La structure organisationnelle était mesurée à partir du nombre de paliers hiérarchiques comme le suggéraient Scholl (1983) et Dieprete (1987). La première étape consistait donc à connaître, à l'aide de questions directes, les postes détenus par les cadres et les services dans lesquels chacun travaillait au moment de l'enquête (finance, personnel, contentieux, informatique, services à l'enfance, aux adultes, aux personnes âgées). La deuxième étape, à l'aide de l'organigramme de chaque CSS, consistait à identifier le nombre de paliers compris dans la voie hiérarchique de laquelle relevait le service et à indiquer pour chaque cadre le nombre de paliers afférents.

La nature du poste occupé était mesurée en posant la question suivante: «Le service dans lequel travaille le cadre est-il généralement considéré dans l'organisation comme 1 = conseil, 2 = hiérarchique ?» Cette question était adaptée de Tremblay et Roger (1991).

Par ailleurs, afin de vérifier rigoureusement les relations exprimées par nos hypothèses qui concernent l'impact de certaines caractéristiques sur le phénomène de plafonnement de carrière, nous avons contrôlé les effets de certaines variables individuelles à savoir l'âge, la scolarité, le sexe, l'ancienneté dans l'organisation et la performance. Cependant, cette dernière variable représentait un problème potentiel. En effet, la nature même de cette variable fait souvent appel à l'appréciation du répondant en l'absence de critères objectifs. Plusieurs auteurs appelés à mesurer la performance ont considéré l'évaluation ou la perception des sujets à l'égard de leur propre performance (Near, 1985; Gerpott et Domsh, 1987; Tremblay et Roger, 1991).

Par contre, Veiga (1981) a constaté une relation positive entre la rémunération et la performance. Considérant que dans les CSS la rémunération 
des cadres est normée et uniforme, et que généralement l'échelle salariale n'est aucunement influencée par la performance, mais strictement par l'ancienneté et l'expérience, nous ne retenons pas cette approche comme mesure de performance. Cependant, la reconnaissance de la contribution exceptionnelle d'un cadre dans son organisation peut amener une rémunération forfaitaire. Cette dernière constituait un indice de performance représentant un indicateur pertinent dans les circonstances. Nous avons donc posé la question suivante: «Au cours du dernier exercice financier où vous avez actualisé le processus de reconnaissance de contributions exceptionnelles, le cadre s'est-il vu reconnaître une contribution exceptionnelle en référence au décret sur la rémunération des cadres?» Le choix de réponse était codé $1=$ oui et $2=$ non.

Le plafonnement de carrière, la variable dépendante de cette recherche, a été mesurée à partir du nombre d'années ou de mois dans le poste actuel à l'instar de plusieurs chercheurs (Veiga, 1981 ; Slocum et al., 1985; Gerpott et Domsh, 1987; Tremblay et Roger, 1991). Comme nous l'avons mentionné précédemment, il n'y a pas de consensus chez les chercheurs quant au seuil délimitant les sujets plafonnés et non plafonnés. Par conséquent, il semblait indiqué de recourir à une approche non basée exclusivement sur la notion de seuil pour classer les sujets réputés ou non en situation de plafonnement de carrière. Comme nous le verrons plus loin, la variable dépendante a été considérée consécutivement comme variable de type continue et de type dichotomique, introduisant dans ce dernier cas, plusieurs seuils. Cette façon de faire a permis d'effectuer des analyses plus poussées du phénomène de plafonnement de carrière sans présumer de l'occurrence d'un seuil.

\section{Résultats}

Afin de vérifier l'existence de relations linéaires entre les différentes variables indépendantes et l'ancienneté dans le poste actuel (la variable dépendante), nous avons utilisé la corrélation de type Pearson. La matrice d'intercorrélations entre les différentes variables présentée au tableau 1 démontre que les coefficients varient entre 0,005 et 0,721 . Notons que les corrélations entre, d'une part, le nombre de paliers hiérarchiques et la taille de l'organisation $(0,721)$ et, d'autre part, l'âge et l'ancienneté $(0,496)$ semblent tout à fait logiques et non surprenantes. En effet, comme l'ont démontré les travaux de Mintzberg (1982), il existe un lien étroit entre la structure organisationnelle et la taille de l'organisation. Par ailleurs, les résultats de recherches antérieures sur le plafonnement de carrière, notamment ceux de Tremblay et Roger (1991), donnent un caractère tout à fait prévisible aux résultats mettant en relation l'âge et l'ancienneté. 
TABleau 1

Matrice d'intercorrélations

\begin{tabular}{|c|c|c|c|c|c|c|c|c|c|c|}
\hline & VARIABLES & 1 & 2 & 3 & 4 & 5 & 6 & 7 & 8 & 9 \\
\hline 1. & Taille de l'organisation & & & & & & & & & \\
\hline 2. & Structure de l'organisation & $0,721 * *$ & & & & & & & & \\
\hline 3. & Nature du poste occupé & 0,005 & $0,256 * *$ & & & & & & & \\
\hline 4. & Âge & $0,160 * *$ & $0,172 * *$ & 0,049 & & & & & & \\
\hline 5. & Sexe & $0,114 * *$ & $0,124 * *$ & 0,016 & 0,018 & & & & & \\
\hline 6. & Scolarité & $-0,036$ & 0,074 & 0,059 & $0,173 * *$ & 0,063 & & & & \\
\hline 7. & Performance & 0,068 & 0,069 & $-0,045$ & 0,109 & 0,010 & $-0,019$ & & & \\
\hline 8. & $\begin{array}{l}\text { Ancienneté } \\
\text { dans l'établissement }\end{array}$ & $0,170 *$ & $0,174 * *$ & 0,036 & $0,496 * *$ & $-0,088$ & $-0,088$ & 0,073 & & \\
\hline 9. & Ancienneté & $-0,017$ & 0,006 & $-0,125 * *$ & $0,378 * *$ & $-0,169 * *$ & $-0,044$ & 0,063 & $0,396 * *$ & \\
\hline
\end{tabular}

** $\mathrm{p}<0,01 \quad * \mathrm{p}<0,05$ 
De plus, la matrice d'intercorrélations présentée au tableau 1 démontre l'absence de corrélation entre, d'une part, l'ancienneté dans le poste actuel et, d'autre part, la taille de l'organisation $(\mathrm{r}=-0,017)$, la structure de l'organisation $(r=-0,006)$, le degré de scolarité $(r=-0,044)$ et finalement la performance $(r=0,063)$.

Par contre, l'âge $(r=0,378, p<0,01)$ et l'ancienneté dans l'établissement $(\mathrm{r}=0,396, \mathrm{p}<0,01)$ sont fortement liés à l'ancienneté dans le poste actuel. Bien que dans une moindre mesure, le sexe $(r=-0,169, p<0,01)$ et la nature du poste occupé $(\mathrm{r}=-0,125, \mathrm{p}<0,01)$ s'avèrent également reliés à l'ancienneté dans le poste actuel.

De prime abord, ces résultats semblent infirmer nos trois hypothèses. En effet, nous constatons d'abord l'absence de corrélation significative entre, d'une part, la taille de l'organisation $(r=-0,017)$, ainsi que la structure de l'organisation $(r=0,006)$ et, d'autre part, l'ancienneté dans le poste actuel. Finalement, on relève la présence d'une relation négative et significative entre la nature du poste occupé et l'ancienneté dans le poste actuel $(\mathrm{r}=-0,125, \mathrm{p}<0,01)$.

Cependant, afin d'éviter d'interpréter faussement des résultats provenant exclusivement d'une analyse univariée, nous avons eu recours à la régression multiple. Le recours à une méthode d'analyse multivariée comme la régression procure une meilleure évaluation des interrelations entre les différentes variables indépendantes. Elle permet de faire ressortir notamment le caractère significatif d'une variable en tenant compte de l'ensemble des variables comprises dans le schéma expérimental (Perrien et al., 1986; Zuccaro, 1992). Le choix de cette méthode a permis d'examiner l'importance de la relation d'association entre les variables indépendantes et l'ancienneté dans le poste actuel, d'en évaluer la signification statistique globale et d'identifier quels facteurs s'avèrent déterminants dans le phénomène de plafonnement de carrière.

Les résultats de la régression multiple, présentés au tableau 2, démontrent que sur huit variables indépendantes, cinq d'entre elles sont significatives soit: l'ancienneté dans l'établissement $(\mathrm{B}=0,196, \mathrm{~T}=5,178, \mathrm{p}<0,01)$, l'âge $(\mathrm{B}=2,59, \mathrm{~T}=5,656, \mathrm{p}<0,01)$, le sexe $(\mathrm{B}=-16,84, \mathrm{~T}=-3,222, \mathrm{p}<0,01)$, la nature du poste $(B=-24,297, T=-3,463, p<0,01)$ et enfin la taille de l'organisation $(B=-0,015, T=-2,075, p<0,05)$. Ces variables expliquent $25 \%$ de la variance de l'ancienneté dans le poste actuel. Pour les besoins de l'analyse, cette variable dépendante est mesurée à ce stade-ci de façon continue, soit en termes de nombre de mois au poste actuel.

De plus, ce tableau présente les résultats d'une deuxième régression effectuée avec contrôle des variables, c'est-à-dire en excluant les variables non significatives lors de la première régression. Ces cinq variables demeurent significatives soit: l'ancienneté dans l'établissement $(B=0,208, T=5,606$, 
$\mathrm{p}<0,01)$, l'âge $(\mathrm{B}=2,456, \mathrm{~T}=5,56, \mathrm{p}<0,01)$, le sexe $(\mathrm{B}=-17,013, \mathrm{~T}=-3,261$, $\mathrm{p}<0,01)$, la nature du poste $(\mathrm{B}=-22,821, \mathrm{~T}=-3,497, \mathrm{p}<0,01)$ et finalement la taille de l'organisation $(B=-0,010, T=-2,106, p<0,05)$ et expliquent toujours $25 \%$ de la variance de l'ancienneté dans le poste actuel.

\section{TABLEAU 2}

Résultats de l'analyse de la régression pour la relation entre certaines variables indépendantes et l'ancienneté dans le poste actuel

\begin{tabular}{|c|c|c|c|c|}
\hline \multirow[t]{2}{*}{ VARIABLES } & \multicolumn{2}{|c|}{$\begin{array}{l}\text { Première régression } \\
\text { sans contrôle }\end{array}$} & \multicolumn{2}{|c|}{$\begin{array}{c}\text { Deuxième régression } \\
\text { avec contrôle }\end{array}$} \\
\hline & B & $\mathbf{T}$ & B & $\mathbf{T}$ \\
\hline Taille de l'organisation & $-0,015$ & $-2,075 *$ & $-0,010$ & $-2,106 *$ \\
\hline Structure de l'organisation & 3,578 & 0,812 & & \\
\hline Nature du poste & $-24,297$ & $-3,463 * *$ & $-22,821$ & $-3,497 * *$ \\
\hline Âge & 2,59 & $5,656 * *$ & 2,456 & $5,56 * *$ \\
\hline Sexe & $-16,84$ & $-3,222 * *$ & $-17,013$ & $-3,261 * *$ \\
\hline Scolarité & $-2,263$ & $-1,392$ & & \\
\hline Performance & 1,518 & 0,277 & & \\
\hline Ancienneté dans l'établissement & 0,196 & $5,178 * *$ & 0,208 & $5,606 * *$ \\
\hline $\mathbf{R}$ & \multicolumn{2}{|c|}{0,5055} & \multicolumn{2}{|c|}{0,5013} \\
\hline $\mathbf{R}^{2}$ & \multicolumn{2}{|c|}{0,2556} & \multicolumn{2}{|c|}{0,2514} \\
\hline $\mathrm{R}^{2}$ ajusté & \multicolumn{2}{|c|}{0,2418} & \multicolumn{2}{|c|}{0,2428} \\
\hline F & \multicolumn{2}{|c|}{$18,544 * *$} & \multicolumn{2}{|c|}{$29,213 * *$} \\
\hline
\end{tabular}

Il convient de souligner que, contrairement à l'approche privilégiée pour obtenir les résultats présentés au tableau 2, plusieurs chercheurs ont mesuré le plafonnement de carrière en considérant cette variable comme étant de type dichotomique, c'est-à-dire en fixant un seuil préétabli afin de déterminer si le sujet est réputé ou non en situation de plafonnement de carrière (Veiga, 1981; Orpen, 1983; Slocum et al., 1985; Near, 1985; Gerpott et Domsh, 1987; Tremblay et Roger, 1991). Cette dernière approche nous semblant plus arbitraire, il paraissait indiqué de compléter notre analyse des résultats en introduisant une gamme de seuils à savoir $60,72,84,133,144$ et 180 mois. Cette approche a permis de vérifier le comportement de nos variables explicatives à différents intervalles. 
TABLEAU 3

Résultats comparatifs des analyses de régression à différents seuils retenus pour le plafonnement de carrière

\begin{tabular}{|c|c|c|c|c|c|c|c|c|c|c|c|c|}
\hline \multicolumn{13}{|c|}{ SEUILS } \\
\hline \multirow{2}{*}{ VARIABLES } & \multicolumn{2}{|c|}{60 MOIS } & \multicolumn{2}{|c|}{72 MOIS } & \multicolumn{2}{|c|}{84 MOIS } & \multicolumn{2}{|c|}{133 MOIS } & \multicolumn{2}{|c|}{144 MOIS } & \multicolumn{2}{|c|}{180 MOIS } \\
\hline & B & $\mathbf{T}$ & B & $\mathbf{T}$ & B & $\mathbf{T}$ & B & $\mathbf{T}$ & B & $\mathbf{T}$ & B & $\mathbf{T}$ \\
\hline $\begin{array}{l}\text { Structure } \\
\text { de l'organisation }\end{array}$ & 0,048 & 1,329 & 0,036 & 1,057 & 0,001 & 0,057 & 0,002 & 0,078 & 0,006 & 0,027 & 0,029 & 1,434 \\
\hline Âge & 0,020 & $5,335 * *$ & 0,019 & $5,447 * *$ & 0,018 & $5,384 * *$ & * 0,015 & $5,106 * *$ & 0,012 & $4,329 * *$ & 0,005 & $2,351 * *$ \\
\hline Sexe & $-0,066$ & $-1,539$ & $-0,068$ & $-1,674$ & $-0,084$ & $-2,168 *$ & $-0,130$ & $-3,724$ & $-0,121$ & $-3,727$ & $-0,108$ & $-4,368 *$ \\
\hline Scolarité & $-0,001$ & $-0,136$ & $-0,008$ & $-0,689$ & $-0,019$ & $-1,646$ & $-0,015$ & $-1,442$ & $-0,010$ & $-1,049$ & $-0,011$ & $-1,526$ \\
\hline Performance & 0,026 & $-2,588$ & $-0,007$ & 0,183 & $-0,046$ & $1,137 *$ & 0,032 & $-0,891$ & $-0,025$ & $-0,759$ & $-0,015$ & $-0,526$ \\
\hline $\begin{array}{l}\text { Ancienneté dans } \\
\text { l'établissement }\end{array}$ & 0,001 & 0,577 & 0,001 & $4,736 * *$ & 0,002 & $4,886 * *$ & 0,000 & 5,535 & 0,001 & $4,251 * *$ & 0,000 & $2,983 * *$ \\
\hline$R^{2}$ ajusté & & 750 & & 2067 & & 233 & & 1777 & & 861 & & 1336 \\
\hline F & 12, & $68 * *$ & & $331 * *$ & & $11 * *$ & & $389 * *$ & & $79 * *$ & & $480 * *$ \\
\hline
\end{tabular}

** $\mathrm{p}<0,01 \quad * \mathrm{p}<0,05$ 
Le tableau 3 illustre les résultats comparatifs des analyses de régression pour différents seuils d'ancienneté dans le poste actuel. On peut constater que ces régressions sont toutes significatives $(\mathrm{p}<0,01)$; les résultats expliquant de 15 à $23 \%$ de la variance de la variable dépendante. De plus, ce tableau permet d'identifier les variables significatives à différents seuils d'ancienneté dans le poste actuel. La structure de l'organisation s'avère constamment non significative à tous les seuils alors que la taille organisationnelle est significative à compter de 144 mois $(\mathrm{p}<0,05)$ et à 180 mois $(\mathrm{p}<0,01)$. La nature du poste occupé devient significative à compter de 72 mois $(\mathrm{p}<0,01)$ dont à 133 mois $(\mathrm{p}<0,05)$. En ce qui a trait aux caractéristiques individuelles, l'âge ressort de façon significative aux seuils de 60 à 144 mois $(\mathrm{p}<0,01)$ et à 180 mois $(\mathrm{p}<0,05)$ tandis que le sexe est significatif à 84 mois $(\mathrm{p}<0,05)$ et de 133 à 180 mois ( $<<0,01$ ). La performance n'est significative qu'au seuil de 84 mois ( $\mathrm{p}<0,05)$, la scolarité n'étant aucunement significative à ces seuils. Finalement, l'ancienneté dans l'établissement, à l'exception du seuil de 60 mois, s'avère constamment significative $(\mathrm{p}<0,01)$.

\section{Discussion}

Le principal objectif de cette étude consistait à identifier les caractéristiques organisationnelles pouvant avoir une influence déterminante sur le plafonnement de carrière des cadres intermédiaires des CSS du Québec.

À cet égard, trois hypothèses ont été formulées. La première, concernant la taille de l'organisation, visait à établir la présence d'une relation négative entre la taille et l'ancienneté dans le poste actuel. La seconde, concernant la structure organisationnelle, visait à vérifier l'impact du nombre de paliers hiérarchiques sur l'ancienneté dans le poste actuel. Finalement, la troisième hypothèse consistait à établir l'absence d'influence de la nature du poste occupé par les cadres sur l'ancienneté dans leur poste actuel. Il convient de rappeler que l'ancienneté dans le poste actuel constituait notre mesure du plafonnement de carrière.

L'hypothèse 1 semble confirmée. En effet, les analyses de régressions multiples (tableau 2) expriment avec constance la présence d'une influence négative et significative. Plus précisément, cette relation indique que plus la taille de l'organisation est grande, moins l'ancienneté dans le poste actuel est élevée. Ces résultats convergent avec ceux de divers auteurs (Dalton et Kesner, 1983 ; Stumpf et Hartman, 1984 ; Grand-Rose et Portwood, 1988) qui constataient globalement que les organisations de grande dimension possèdent habituellement des systèmes plus formalisés de gestion des carrières et de formation, ce qui améliore la mobilité et la flexibilité de carrière. Nos résultats 
corroborent également ceux de Near (1985) qui avait constaté que le plafonnement de carrière survenait plus fréquemment dans les organisations de dimension moins importante.

En outre, bien que nous n'ayons pu le vérifier empiriquement, le contexte socio-économique prévalant dans certaines régions périphériques peut également constituer un facteur explicatif du phénomène de plafonnement de carrière. C'est le cas notamment pour des régions périphériques où l'on retrouve les CSS de plus petite dimension (moins de 200 postes). En effet, le marché de l'emploi, comme les coûts et les différents impacts d'une relocalisation, comptent parmi les facteurs incitant à une certaine stabilité. En résumé, nous avons établi que la taille organisationnelle influençait significativement l'ancienneté dans le poste actuel. On peut donc inférer que les probabilités de plafonnement de carrière sont plus fortes chez les cadres travaillant dans des organisations à dimension moins importante.

Selon l'hypothèse 2, il existait une relation négative et significative entre la structure organisationnelle (nombre de paliers hiérarchiques compris dans le service) et l'ancienneté dans le poste actuel; elle est infirmée. En effet, comme mentionné précédemment, les résultats relatifs à cette variable s'avèrent constamment non significatifs à travers les différentes analyses effectuées, à savoir les régressions multiples intégrant la variable dépendante mesurée de façon continue ou dichotomique (tableaux 2 et 3 ). Ces résultats divergent des suggestions exprimées par certains auteurs, selon lesquels un cadre travaillant dans un service comportant peu de paliers hiérarchiques bénéficierait de moins de possibilités d'avancement et serait, par conséquent, plus susceptible de vivre une situation de plafonnement de carrière (Spilerman, 1977; Vardi, 1980; Tremblay, 1991).

Ces résultats apparemment surprenants pourraient s'expliquer par le fait que les structures organisationnelles des CSS comprennent entre 2 et 5 paliers hiérarchiques; de plus, $98 \%$ des répondants œuvrent dans des services comportant de 3 à 5 paliers. Il s'agit donc d'une population homogène où l'on retrouve une très faible variance quant au nombre de paliers hiérarchiques. Aussi, bien que la forme relativement aplatie des structures organisationnelles des CSS puisse constituer un frein à l'avancement (mobilité verticale ascendante), à cause notamment du phénomène de goulot d'étranglement (Spilerman, 1977; Vardi, 1980), elle n'en constitue pas pour autant un frein aux autres types de mobilité verticale (rétrogradations) et horizontale (mutations). Il est possible que plusieurs répondants aient expérimenté ces deux derniers types de mobilité, ce qui a nécessairement eu une influence sur notre variable dépendante qui se mesurait à partir de l'ancienneté au poste actuel. Sur le plan statistique, il est également possible que le phénomène de colinéarité entre la structure et la taille (tableau 1) explique la relation non significative entre la structure organisationnelle et l'ancienneté dans le poste. 
La troisième et dernière hypothèse de cette recherche suggérait l'absence de différence significative du niveau d'ancienneté dans le poste actuel entre les cadres travaillant dans un service-conseil comparativement à ceux qui occupaient un poste dans un service hiérarchique. Les résultats tendent à infirmer cette hypothèse. En effet, nous avons constaté la présence d'une relation négative et significative entre la nature du poste occupé et l'ancienneté dans le poste actuel $(B=-22,821, T=-3,497, p<0,01)$, cette relation étant confirmée dans la régression multiple avec contrôle.

Ceci signifie donc que, contrairement à ce qu'avaient rapporté Tremblay et Roger (1991), les cadres œuvrant dans un service-conseil ont une plus forte propension à accumuler de l'ancienneté dans leur poste et par le fait même, deviennent plus sujets au plafonnement de carrière que leurs homologues travaillant dans un service hiérarchique. Ces résultats pourraient s'expliquer par le phénomène de cloisonnement des emplois tel qu'énoncé par Spilerman (1977). En effet, comme les services-conseils des CSS regroupent généralement des professionnels aussi différents que des avocats, comptables, informaticiens, spécialistes en gestion de ressources humaines, le passage d'une filière à l'autre peut être plus difficile que pour les cadres des services hiérarchiques qui se caractérisent par une plus forte homogénéité disciplinaire.

Par contre, une étude a fait ressortir que les cadres quittent généralement l'organisation lorsqu'ils constatent qu'ils se retrouvent dans une filière d'emploi avec peu de perspectives d'avancement, comme c'est le cas pour certains services-conseils (Scholl, 1983). Or, c'est le contraire qui semble se produire chez les sujets de notre étude. Ce phénomène peut s'expliquer par le fait que les cadres évoluant dans les secteurs publics et parapublics sont, dans une certaine mesure, captifs de ces secteurs organisationnels. La faible fréquence de mobilité interétablissements, de même que les préjugés persistants à l'égard des secteurs publics et parapublics qui freineraient cette mobilité, nous apparaissent comme des motifs plausibles. D'ailleurs, cette faible propension des cadres québécois à changer de secteur organisationnel a déjà fait l'objet d'une vérification empirique (Fabi, 1984). Cette étude révélait, chez un échantillon de diplômés M.B.A., une très forte tendance à se maintenir dans le secteur organisationnel du début de carrière et cela, aussi bien chez les cadres du secteur privé que chez leurs collègues des secteurs publics et parapublics.

En somme, ces résultats convergent avec la littérature concernant la mobilité, selon laquelle certains postes, de par leur nature ou leur secteur, affichent un caractère distinct, ce qui limite les possibilités de mobilité pour les gens qui les occupent en raison, notamment, du phénomène de cloisonnement interservices ou interfonctions. Nos résultats contribuent donc à démontrer l'existence d'une relation significative entre la nature du poste occupé et le phénomène de plafonnement de carrière. 
L'approche utilisée pour mesurer le plafonnement de carrière revêt, à notre avis, une importance capitale compte tenu de son influence sur l'analyse et l'interprétation des résultats obtenus. À cet égard, le tableau 3 illustre le danger d'effectuer des analyses du plafonnement de carrière en fonction d'un seuil préétabli et mesuré comme une variable de type dichotomique. En effet, nous constatons, à la lecture des résultats qui y sont présentés, le potentiel d'erreurs d'interprétation qu'introduit l'approche dichotomique, particulièrement, en ce qui a trait à la taille organisationnelle, à la nature du poste occupé et au sexe.

D'une part, les seuils couramment utilisés par les chercheurs, à savoir 60 , 72,84 et 133 mois ne permettent pas de déceler une quelconque influence de la taille organisationnelle sur le plafonnement de carrière, alors qu'à compter de 144 mois cette variable s'avère significative. D'autre part, si nous avions choisi de retenir 60 mois comme seuil critique, nous aurions conclu, comme avec le sexe, à l'absence d'influence significative de la nature du poste occupé sur l'ancienneté dans le poste actuel. Un résultat lourd de conséquence: il porterait atteinte à l'amélioration de la connaissance du phénomène de plafonnement de carrière, considérant l'influence significative de ces variables à compter de 72 mois.

Finalement, compte tenu de l'importance accordée aux ressources humaines et à leur développement, on peut espérer que ces résultats inciteront les organisations à élaborer des stratégies permettant de gérer efficacement le phénomène croissant du plafonnement de carrière, comme l'a suggéré Tremblay (1992). De plus, les structures organisationnelles devraient être revues en fonction d'une conception du travail moins axée sur la spécialisation, réduisant ainsi le cloisonnement entre les filières d'emplois et permettant de susciter plus d'occasions de mobilité. D'ailleurs, des structures assouplies et plus horizontales favorisent une plus grande responsabilisation et une meilleure distribution du pouvoir, autant de facteurs contribuant à accroître la valorisation dans un poste donné (Cardinal et Lamoureux, 1992 ; Tremblay, 1992). Une conception renouvelée du succès de carrière, moins axée exclusivement sur l'avancement, valorisant aussi les autres types de mobilité et les réalisations à l'intérieur d'un même poste, reflétera un changement de valeurs à l'égard du travail et l'émergence d'une nouvelle culture de gestion des ressources humaines (Cardinal et Lamoureux, 1992). Cette gestion renouvelée des carrières (Guérin et Wils, 1992) nous semble particulièrement importante dans les PME constamment aux prises avec l'épineux problème de conservation de leurs ressources humaines spécialisées. Or, une gestion innovatrice permettrait l'actualisation de leur potentiel à travers l'enrichissement des emplois, ce qui pourrait remplacer l'approche traditionnelle de l'avancement hiérarchique dans des filières promotionnelles établies. On se situe donc ici dans la perspective du design flexible des emplois qui croissent avec l'amélioration des compétences des titulaires. Il va 
sans dire qu'une telle orientation appelle des pratiques de formation continue associées à des pratiques de rémunération des compétences et des connaissances plutôt que du niveau hiérarchique des emplois. De telles modifications risquent de provoquer certaines résistances chez les dirigeants de PME. Mais ces derniers devraient y voir une façon innovatrice de réagir à l'attrait exercé par les grandes organisations auprès de leurs ressources humaines les plus convoitées.

\section{Conclusion}

La présente recherche repose fondamentalement sur l'interrogation suivante: quels sont les impacts de certaines caractéristiques organisationnelles sur le plafonnement de carrière ? Pour y répondre de façon satisfaisante, nous avons procédé à une étude empirique auprès de 441 cadres intermédiaires provenant des 14 centres de services sociaux du Québec. Les résultats révèlent d'abord que la taille organisationnelle semble avoir une influence négative sur le phénomène de plafonnement de carrière. Il ressort en effet que les répondants œuvrant au sein des organisations de dimension moins importante sont plus sujets au plafonnement de carrière que leurs collègues travaillant dans des organisations regroupant un plus grand nombre d'employés. Quant aux autres déterminants organisationnels, les résultats font ressortir l'absence d'influence significative de la structure organisationnelle sur le plafonnement de carrière, ce dernier phénomène s'avérant par ailleurs influencé par la nature du poste occupé. À cet effet, on relève que les cadres d'un service-conseil semblent plus sujets au plafonnement de carrière que leurs collègues des services hiérarchiques. Finalement, bien qu'elles n'aient été considérées qu'à titre de variables de contrôle, certaines caractéristiques individuelles se sont révélées des déterminants significatifs du plafonnement de carrière, à savoir l'âge, l'ancienneté dans l'organisation ainsi que le sexe. Ainsi, contrairement à certaines observations antérieures, il ressort que les hommes semblent plus sujets au plafonnement de carrière que leurs collègues féminins.

Une autre contribution importante de cette étude réside dans la démonstration empirique des dangers inhérents à l'approche traditionnelle basée sur le recours exclusif à la notion de seuil d'ancienneté au poste actuel pour classer les cadres réputés en situation de plafonnement de carrière. Il ressort en effet que l'utilisation de tels seuils fixés arbitrairement pourraient conduire les chercheurs à de fausses interprétations. C'est ainsi que nos résultats ont permis de faire ressortir l'influence significative de la taille organisationnelle sur le plafonnement de carrière, ces analyses étant basées sur une mesure continue de la variable dépendante. Pourtant, le recours exclusif à une mesure dichotomique de cette variable, conformément aux divers seuils traditionnellement retenus, 
nous aurait amené à une conclusion opposée. Il s'agit là d'un enseignement qui devrait éclairer les recherches ultérieures dans le domaine.

La prudence scientifique impose toutefois la formulation de certaines mises en garde à l'égard des résultats obtenus dans le cadre de la présente recherche. La portée de ces résultats est en effet restreinte par des limites de nature méthodologique et conceptuelle. La première limite concerne la validité externe. L'échantillonnage, bien qu'impliquant une population de cadres intermédiaires, fut réalisé dans un réseau spécifique d'organisations du secteur public. Aussi, ces résultats ne peuvent être généralisés qu'avec circonspection à des PME privées ou à d'autres types d'organisations. Par ailleurs, cette étude s'inscrit dans le courant objectif de recherche qui se caractérise par la mesure quantitative de l'ancienneté au poste actuel. Ce faisant, nous avons nécessairement négligé l'approche plus subjective du plafonnement de carrière qui se concentre sur les attitudes et les perceptions engendrées par ce phénomène.

Dans le prolongement de cette étude, il serait pertinent d'améliorer notre modèle heuristique du plafonnement de carrière en faisant intervenir des variables modératrices. On pourrait, par exemple, introduire certaines caractéristiques individuelles et organisationnelles afin de vérifier leur impact sur la relation entre diverses variables indépendantes et le plafonnement de carrière. $\mathrm{Au}$ plan de la gestion, nos résultats font ressortir la pertinence de revoir la conception traditionnelle voulant que le succès et l'épanouissement d'une carrière administrative se mesure exclusivement à partir de la progression dans la hiérarchie organisationnelle. Une conception renouvelée exigera un changement au niveau des attitudes et des pratiques de gestion des carrières. À cet égard, on doit envisager une valorisation des différents types de mobilité latérale autres que l'avancement, ainsi que des interventions de formation et de stimulation des personnes à l'intérieur de leur poste actuel. Il s'agit là d'un défi important pour les PME désireuses de conserver et de motiver leurs ressources humaines, en particulier, les plus qualifiées et les plus ambitieuses. 


\section{Bibliographie}

ANDERSON, J.C., G.T. MiLkoviCH et A. TSUI (1981), «A model of intra-organizational mobility », Academy of Management Review, vol. 6, $\mathrm{n}^{\circ} 4$, p. 529-538.

AUDET, M. (1990), «La fonction ressources humaines a un avenir», Gestion, vol. 15, $\mathrm{n}^{\circ} 1$, p. 17-22.

BEAUCHAMP, S. et B. FABI (1992), «Les déterminants organisationnels du plafonnement de carrière : étude empirique réalisée auprès d'une population de cadres intermédiaires des CSS du Québec », Info Ressources Humaines, vol. 16, $\mathrm{n}^{\circ}$ 1, p. 12-16.

BEAUSOlEIL, G. (1987), «Intervention socio-économique de l'État: problèmes et perspective », Commission d'enquête sur les Services de Santé et de Services Sociaux, Québec, Publications du Québec.

BEEHR, T.A. et D.L. JUNTUNEN (1990), «Promotions and employees perceived mobility channels : the effects of employee sex, employee group, and initial placement», Human Relations, vol. 43, $\mathrm{n}^{\circ}$ 5, p. 455-472.

BEGNOCHE-SMITH, C.B. (1979), «Influence of internal mobility opportunity structure and set of worker turnover pattern », Administrative Sciences Quarterly, 24, p. $362-381$.

BourbonNAIS, J.P. et A. Gosselin (1988), «Les défis de la gestion des ressources humaines pour les années 90: un tour d'horizon», Gestion, vol. 13, n 1 , p. 23-29.

CARDinAL, L. et C. LAMOUREUX (1992), «Le plateau de carrière chez les gestionnaires : diagnostic et intervention », Gestion, vol. 17, $\mathrm{n}^{\circ} 3$, p. 83-91.

CHAO, G.T. (1990), «Exploration of the conception and measurement of career plateau : a comparative analysis », Journal of Management, vol. 16, $n^{\circ} 1$, p. 181-193.

ChILDS, A. et R.J. KLIMOSKI (1986), «Successfully predicting career success : an application of the biographical inventory », Journal of Applied Psychology, vol. $71, \mathrm{n}^{\circ} 1$, p. 3-8.

DALTON, D.R. et I.F. KESNER (1983), «Inside/outside succession and organizational size: the pragmatics of executive replacement », Academy of Management Journal, vol. $26, \mathrm{n}^{\circ} 4$, p. 736-742.

DIPRETE, T.M. (1987), « Horizontal and vertical mobility in organization », Administrative Science Quarterly, vol. 32, septembre, p. 422-444.

FABI, B. (1984), «Privé ou public: choix et transfert de secteur organisationnel », Relations industrielles, vol. $39, \mathrm{n}^{\circ} 2, \mathrm{p} .313-331$.

FABI, B. (1991), «Les facteurs de contingence des cercles de qualité : une synthèse de la documentation empirique ", Canadian Journal of Administrative Sciences, vol. 8, n 3 , p. 161-174. 
FABI, B. (1992), «Success factors in quality circles : a review of empirical evidence», International Journal of Quality and Reliability Management, vol. 9, $\mathrm{n}^{\circ} 2$, p. 16-31.

FIELDMAN, D.C. et B.A. WEITZ (1988), «Career plateaus reconsidered», Journal of Management, vol. 14, $\mathrm{n}^{\circ} 1$, p. 69-80.

GERPOTT, T.J. et M. DOMSCH (1987), «R \& D professionals reactions to the career plateau : mediating effects of supervisory behaviours and job characteristics », $R \& D$ Management, vol. $17, \mathrm{n}^{\circ} 2$, p. 103-117.

Gould, S. et L.E. PENLEY (1985), «A study of the correlates of the willingness to relocate », Academy of Management Journal, vol. 28, $\mathrm{n}^{\circ} 2$, p. 472-478.

Gould, S. et L.E. PENLEY (1984), « Career strategies and salary progression: a study of their relationships in a municipal bureaucracy», Organizational Behavior and Human Performance, vol. 34, p. 244-265.

GRAND-Rose, C.S. et J.D. PORTwOod (1988), « Matching individual career plans and organizational career management », Academy of Management Journal, vol. 31, $\mathrm{n}^{\circ} 4$, p. 699-719.

GRANDJEAN, B.D. (1981), « History and career in bureaucratic labor market », American Journal of Sociology, vol. 86, $\mathrm{n}^{\circ}$ 5, p. 1057-1093.

GUÉRIN, G. et T. WILS (1992), «La gestion des carrières: une typologie des pratiques », Gestion, vol. 17, $\mathrm{n}^{\circ} 3$, p. 48-63.

HALL, D.T. (1985), «Project work as an antidote to career plateauing in the declining engineering organization», Human Ressource Management, vol. 24, $\mathrm{n}^{\circ} 3$, p. 271-292.

MILLS, Q.D. (1985), «Seniority vs ability in promotion decisions », Industrial and Labor Relations Review, vol. 38, $\mathrm{n}^{\circ} 3$, p. 421-425.

MinTZBERG, H. (1982), Structure et dynamique des organisations, Paris, Éditions d'Organisation.

NEAR, J.P. (1985), «A discriminant analysis of plateaued versus nonplateaued managers », Journal of Vocational Behavior, vol. 26, p. 177-188.

NYSTROM, P.C. (1986), « Comparing beliefs of line technostructure managers », Academy of Management Journal, vol. 29, $\mathrm{n}^{\circ} 4$, p. 812-819.

PERRIEN, J., J.E. ChÉRON et M. ZINS (1986), Recherche en Marketing : méthodes et décisions, Montréal, Gaëtan Morin.

Rochon, J. (1988), «Rapport de la Commission d'enquête sur les Services de Santé et de Services Sociaux », Québec, Publications du Québec.

SAVERY, L.K. (1990), «Managing plateau employees », Management Decision, vol. 28, $\mathrm{n}^{\circ} 3$, p. $46-50$. 
SCHOLL, R. (1983), «Career line and employment stability », Academy of Management Journal, vol. $26, \mathrm{n}^{\circ} 1$, p. 86-103.

Slocum, J.W., W.L. CRON, R.W. HANSEN et S. RAWLINGS (1985), «Business strategy and the management of plateaued employees», Academy of Management Journal, vol. $28, \mathrm{n}^{\circ} 1, \mathrm{p} .133-154$.

SPILERMAN, C. (1977), « Career, labor market structure and socioeconomic achievement, American Journal of Sociology, vol. 83, $\mathrm{n}^{\circ} 3$, p. 551-593.

STUMPF, Stephen A. et K. HARTMAN (1984), «Individual exploration to organizational commitment or withdrawal, Academy of Management Journal, vol. 27, $\mathrm{n}^{\circ} 2$, p. 308-327.

SYLVIA, R. et K.M. SYLVIA (1986), "An empirical investigation of the impacts of career plateauing», International Journal of Public Administration, vol. 8, $\mathrm{n}^{\circ} 3$, p. 227-241.

Tremblay, M. (1992), «Comment gérer le blocage des carrières », Gestion, vol. 17, $\mathrm{n}^{\circ} 3$, p. 73-82.

Tremblay, M. et A. Roger (1991), «Stabilité dans l'emploi et le plafonnement de carrière », Étude empirique des variables explicatives de la mobilité interne auprès d'une population de cadres canadiens, France, Institut d'administration des entreprises.

TREMBLAY, M. et A. Roger (1992), «La préparation de la relève dans les entreprises », Gestion, vol. 17, n 3, p. 64-72.

VARDI, Y. (1980), «Organizational career mobility: an integrative model », Academy of Management Review, vol. 5, $\mathrm{n}^{\circ}$ 3, p. 341-355.

VEIGA, J.F. (1981), «Plateaued versus nonplateaued managers: career patterns, attitudes, and path potential », Academy of Management Journal, vol. 24, $\mathrm{n}^{\circ} 3$, p. 566-578.

WARREN, E.K., J.A.F. STONER et T.P. FERENCE (1977), « Managing the career plateau », Academy of Management Review, vol. 2, $\mathrm{n}^{\circ} 3$, p. 602-612.

ZUCCARO, C. (1992), «Mallows' Cp statistic and model selection in multiple linear regression », Journal of the Market Research Society, vol. 34, ${ }^{\circ} 2$, p. 163-172. 